\title{
- Excited charm meson spectroscopy from B decays at
} 2 LHCb

\section{R.I. Rabadan Trejo ${ }^{a, *}$}

$4 \quad$ On behalf of the LHCb collaboration

$5{ }^{a}$ Aix-Marseille Universite, CNRS/IN2P3, CPPM,

$6 \quad 13288$ Marseille, France

$7 \quad$ E-mail: raul.iraq.rabadan.trejo@cern.ch

Recent charm meson spectroscopy results from amplitude analysis of $B$ decays to open charm states at $\mathrm{LHCb}$ are reported. The amplitude analyses technique is used in the description of $B^{-} \rightarrow D^{*+} \pi^{-} \pi^{-}$decays and a study of the excited charm meson spectra in the $D^{*+} \pi^{-}$system is performed.

40th International Conference on High Energy physics - ICHEP2020

July 28 - August 6, 2020

Prague, Czech Republic (virtual meeting)

\footnotetext{
${ }^{*}$ Speaker
} 
10

\section{Introduction}

The quark model has been successful in predicting the charm meson spectra. A recent computation of the properties of excited charm mesons is given in Ref. [1]. Charm meson spectroscopy regained considerable attention after the discovery of several excited charm and charm-strange states in inclusive reactions by the BABAR [2,3] and the LHCb experiments [4, 5]. Measurements of the properties of the new states provide important benchmarks for the theoretical predictions. Moreover, to make quark model spectroscopy classifications, their quantum numbers need to be determined. Amplitude analyses of exclusive B decays to final-states with an excited charm meson allow for a full spin-parity analysis of these states. The LHCb experiment has performed numerous amplitude analyses to investigate the $D_{J}$ spectroscopy in the $D \pi$ system [6-9]. For instance, the quantum numbers of the $D_{3}^{*}(2750)$ meson were measured in $B^{0} \rightarrow \bar{D}^{0} \pi^{+} \pi^{-}$and $B^{-} \rightarrow D^{+} \pi^{-} \pi^{-}$decays. For other states, namely the $D_{0}(2550), D_{J}^{*}(2600)$ and $D(2740)$ [10] the $J^{P}$ assignments remain inconclusive. For this task, additional studies of the $D^{*} \pi$ system in $B$ meson decays are necessary since they give access to both natural and unnatural spin-parity states (except for the $J^{P}=0^{-}$). It is therefore important to continue exploring the charm-meson spectroscopy in exclusive $B$-decays in order to unambiguously identify new states and compare their measured properties against theory predictions. This proceeding reports on the latest results on excited charm-meson spectroscopy from amplitude analyses of $B$ decays at $\mathrm{LHCb}$.

\section{Recent results on $D_{J}$ spectroscopy from $B^{-} \rightarrow D^{*+} \pi^{-} \pi^{-}$decays}

A study of the $D_{J}$ spectroscopy in the $D^{*+} \pi^{-}$system is carried out through an amplitude analysis of $B^{-} \rightarrow D^{*+} \pi^{-} \pi^{-}$[11] decays. The analysis is based on a data sample corresponding to a total integrated luminosity of $4.7 \mathrm{fb}^{-1}$ of $p p$ collisions collected at a center-of-mass energies of 7 , 8 and $13 \mathrm{TeV}$ with the $\mathrm{LHCb}$ detector. The $B^{-} \rightarrow D^{*+} \pi^{-} \pi^{-}$candidates are reconstructed using the decay chain $D^{*+} \rightarrow D^{0} \pi^{+}$and $D^{0} \rightarrow K^{-} \pi^{+}$. A multivariate selection based on the kinematic and topological information of the decay is employed in order to reduce the combinatorial background. Figure 1a shows the $m\left(D^{*+} \pi^{-} \pi^{-}\right)$invariant mass distribution for the selected candidates.

The amplitude analysis is performed on candidates within a $2 \sigma$ window centered around the $B^{-}$mass, where $\sigma=17 \mathrm{MeV}$. A total of 79120 signal events are available within the signal window. The Dalitz plot of the $B^{-} \rightarrow D^{*+} \pi^{-} \pi^{-}$candidates is shown in Fig. 1b. The decay mode contains two indistinguishable pions in the final state, giving two $D^{*+}$ mass combinations. Therefore, the $B^{-}$ Dalitz plot is described as a function of $m^{2}\left(D^{*+} \pi^{-}\right)_{\min }$ and $m^{2}\left(D^{*+} \pi^{-}\right)_{\max }$, the lower and higher values of the two pair combinations. The resonances included in the model used to describe the decay are listed in Tab. 1. The best fit to data is obtained using a model where the $J^{P}=1^{+} S$ and $J^{P}=0^{-}$contributions are described by quasi model independent (QMI) amplitudes [12, 13] while other contributions are described using relativistic Breit-Wigner functions. The fit projection onto $m\left(D^{*+} \pi^{-}\right)_{\text {min }}$ is shown in Fig. 2b. The fitted resonances parameters are given in Tab. 1. To extract the parameters of the $D_{1}(2430)$ state, a fit is performed with the QMI description of the $J^{P}=1^{+} S$ amplitude replaced by a Breit-Wigner function. Similarly, the $D_{0}(2550)$ parameters are measured by replacing the $J^{P}=0^{-}$amplitude with a Breit-Wigner function. Details on the fitting procedure and the obtained fractional contributions and phases can be found in Ref. [11]. 

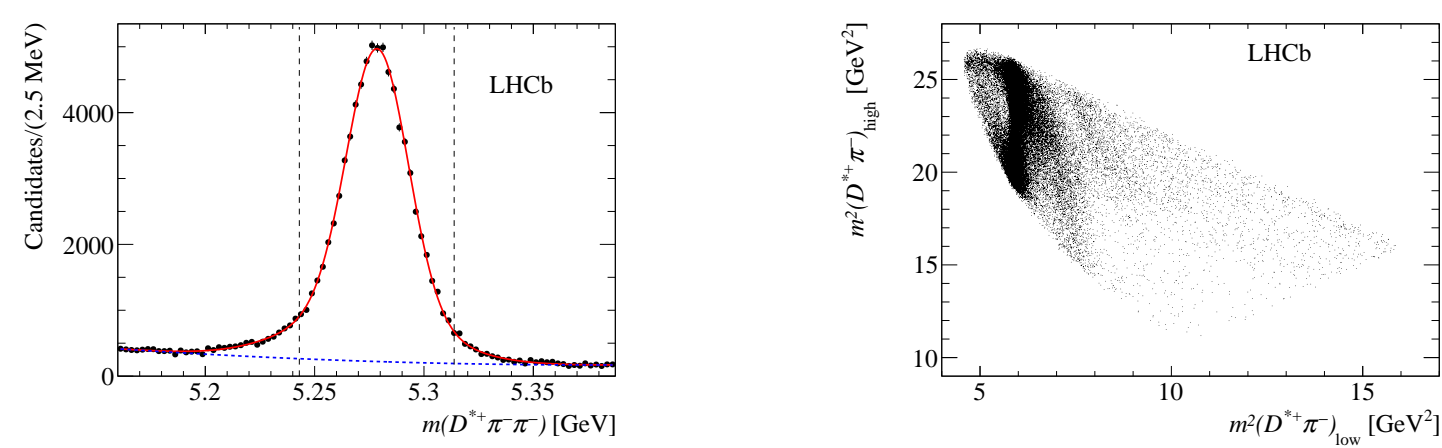

Figure 1: Left plot shows the invariant mass distribution for $B^{-} \rightarrow D^{*+} \pi^{-} \pi^{-}$candidates after selection. The vertical dashed lines indicate the signal region. The Dalitz plot distribution are shown in the plot on the right.
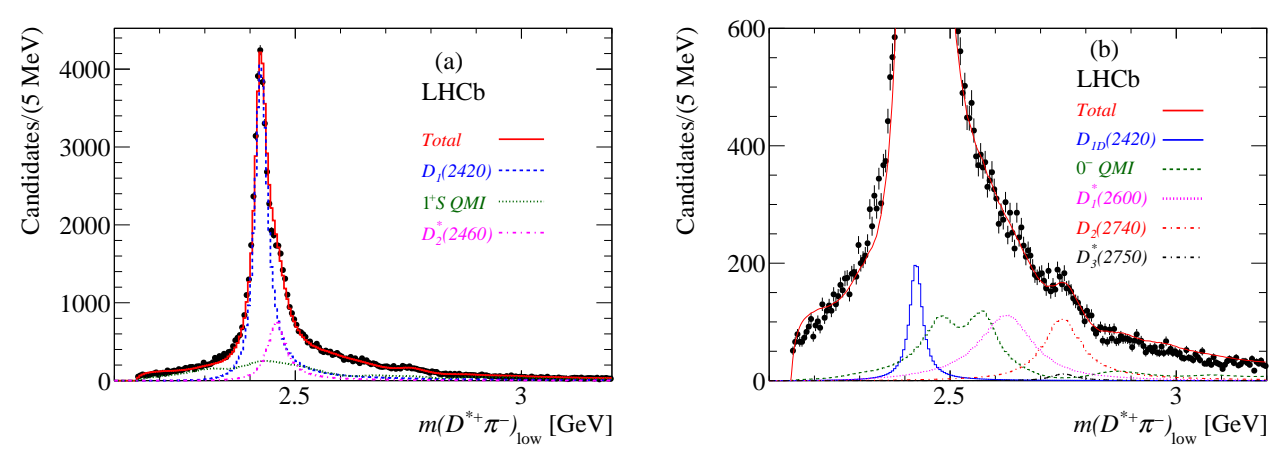

Figure 2: Projection of the data and amplitude fit onto $m\left(D^{*+} \pi^{-}\right)_{l o w}$ for $B^{-} \rightarrow D^{*+} \pi^{-} \pi^{-}$candidates. Left plot shows the same $m\left(D^{*+} \pi^{-}\right)_{l o w}$ projection, but zoomed in.

Searches for additional states are performed for the most significant amplitudes: $J^{P}=1^{-}$, $J^{P}=1^{+} D$ and $J^{P}=2^{+}$. No evidence for additional contributions is found. For the less significant contributions, i.e. the $D_{2}(2740)$ and $D_{3}^{*}(2750)$, Breit-Wigner line shapes are used and extra fits with different spin-parity assignments are performed. Alternative hypotheses are tried for both states but excluded with significances greater than $8 \sigma$. In conclusion, the quantum numbers for the $D_{0}(2550)$ and $D_{1}^{*}(2600), D_{1}(2420), D_{2}(2740)$ and $D_{3}^{*}(2750)$ are determined.

Finally, the mixing between the $D_{1}(2420)$ and $D_{1}(2430)$ is also investigated. Mixing can occur via the $D^{*} \pi$ channel, the resulting $D_{1}^{\prime}$ and $D_{1}$ amplitudes being a superposition of the $S-$ and $D$-wave amplitudes

$$
\begin{aligned}
& A^{D_{1}^{\prime}}=A^{1 S} \cos \omega-A^{1 D} \sin \omega e^{i \psi}, \\
& A^{D_{1}}=A^{1 S} \sin \omega+A^{1 D} \cos \omega e^{-i \psi},
\end{aligned}
$$

where $\omega$ is the mixing angle and $\psi$ is a complex phase. In the model used to extract the mixing parameters, the $J^{P}=1^{+}$states are described by relativistic Breit-Wigner functions while the $J^{P}=0^{-}$ amplitude is described with a QMI approach. All other resonances are parametrized in terms of Breit-Wigner functions with parameters fixed to the values given in Tab. 1. The measured $D_{1}$ and 
Table 1: List of resonances contributing to the $B^{-} \rightarrow D^{*+} \pi^{-} \pi^{-}$decay. The resonance parameters obtained from the amplitude analysis are given.

\begin{tabular}{|c|c|c|c|c|c|}
\hline Resonance & $J^{P}$ & \multicolumn{2}{|c|}{ Mass [MeV] } & Width [MeV] & Significance $(\sigma)$ \\
\hline$D_{1}(2420)$ & $1^{+}$ & $2424.8 \pm 0.1$ & \pm 0.7 & $33.6 \pm 0.3 \pm 2.7$ & \\
\hline$D_{1}(2430)$ & $1^{+}$ & $2411 \pm 3$ & \pm 9 & $309 \pm 9 \pm 28$ & \\
\hline$D_{2}^{*}(2460)$ & $2^{+}$ & $2460.56 \pm 0.35$ & & $47.5 \pm 1.1$ & \\
\hline$D_{0}(2550)$ & $0^{-}$ & $2518 \pm 2$ & \pm 7 & $199 \pm 5 \pm 17$ & 53 \\
\hline$D_{1}^{*}(2600)$ & $1^{-}$ & $2641.9 \pm 1.8$ & \pm 4.5 & $149 \pm 4 \pm 20$ & 60 \\
\hline$D_{2}(2740)$ & $2^{-}$ & $2751 \pm 3$ & \pm 7 & $102 \pm 6 \pm 26$ & 16 \\
\hline$D_{3}^{*}(2750)$ & $3^{-}$ & $2753 \pm 4$ & \pm 6 & $66 \pm 10 \pm 14$ & 8.7 \\
\hline$D_{1}$ & $1^{+}$ & $2423.7 \pm 0.1$ & \pm 0.8 & $31.5 \pm 0.1 \pm 2.1$ & \\
\hline$D_{1}^{\prime}$ & $1^{+}$ & $2452 \pm 4$ & \pm 15 & $444 \pm 11 \pm 36$ & \\
\hline
\end{tabular}

$D_{1}^{\prime}$ are reported in Tab. 1 . The mixing angle is measured to be $\omega=-0.063 \pm 0.019 \pm 0.004$ which is consistent with previous results [14].

\section{3. $D_{s J}$ spectroscopy}

An amplitude analysis of $B_{s}^{0} \rightarrow \bar{D}^{0} K^{-} \pi^{+}$decays is performed based on a data sample corresponding to an integrated luminosity of $3.0 \mathrm{fb}^{-1}$ of pp collisions collected by the LHCb detector $[15,16]$. The signal yield available (11 $300 \pm 160)$, allows for a careful examination of the $D_{s J}$ spectroscopy in the $\bar{D}^{0} K^{-}$system. The data is described using an amplitude model containing 6 $K^{-} \pi^{+}$resonances, $4 \bar{D}^{0} K^{-}$resonances, 3 virtual resonances, and a nonresonant component (see Ref. $[15,16]$ for details on the model). Figure 3 shows the fit projections onto $m\left(\bar{D}^{0} K^{-}\right)$. The spin of the previously observed $D_{s 2}^{*}(2573)^{-}$is confirmed to be 2 and its mass parameters are measured. The results from the amplitude analysis include the first observation and quantum number determination of the $D_{s 1}^{*}(2860)^{-}$and $D_{s 3}^{*}(2860)^{-}$mesons. The full results of the amplitude analysis including the measured mass and widths for the three excited charm-strange mesons are reported in Ref. $[15,16]$.
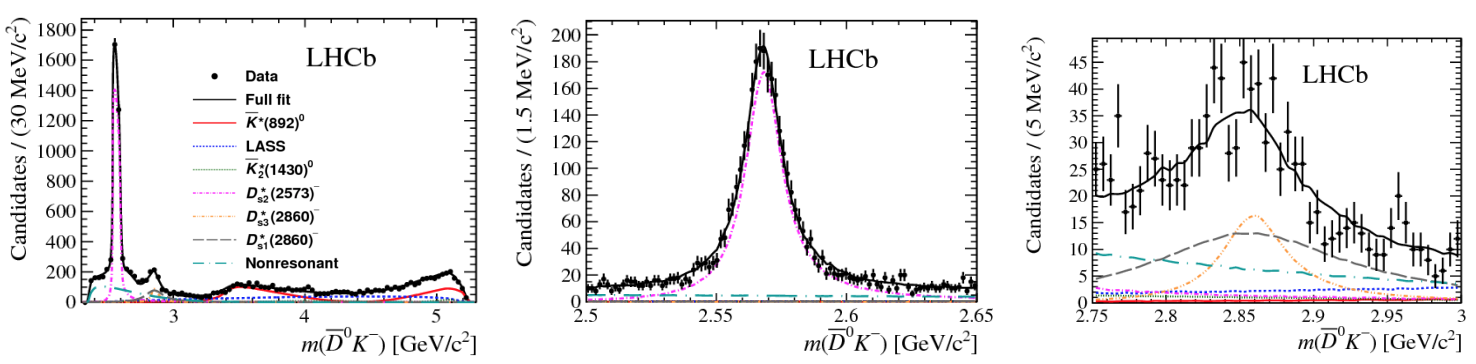

Figure 3: Projection of the data and amplitude fit onto $m\left(\bar{D}^{0} K^{-}\right)$(left) with zooms around (middle) the $D_{s 2}^{*}(2573)^{-}$resonance and (right) the $D_{s 1}^{*}(2860)^{-}$region. 


\section{Summary}

The LHCb experiment has extensively exploited the amplitude analysis technique to investigate the excited charm meson spectroscopy from $B$-meson decays using the data collected during the LHC Run 1. With the large datasets collected by the LHCb during Run 2, we will continue gaining additional knowledge on the $D_{J}$ and $D_{S J}$ meson spectroscopy from fully hadronic $B$ decays with production of excited charm and charm-strange states.

\section{References}

[1] S. Godfrey and K. Moats, Properties of excited charm and charm-strange mesons, Phys. Rev. D 93 (2016) 034035.

[2] BABAR collaboration, Observation of new resonances decaying to $D \pi$ and $D^{*} \pi$ in inclusive $e^{+} e^{-}$collisions near $\sqrt{s}=10.58 \mathrm{GeV}$, Phys. Rev. D 82 (2010) 111101.

[3] BABAR collaboration, Study of $D_{s J}$ decays to $D^{*} K$ in inclusive $e^{+} e^{-}$interactions, Phys. Rev. D 80 (2009) 092003.

[4] LHCв collaboration, Study of $D_{J}$ meson decays to $D^{+} \pi^{-}, D^{0} \pi^{+}$and $D^{*+} \pi^{-}$final states in $p p$ collisions, JHEP 1309 (2013) 145.

[5] LHCв collaboration, Study of $D_{S J}$ decays to $D^{+} K_{\mathrm{S}}^{0}$ and $D^{0} K^{+}$final states in pp collisions, JHEP 1210 (2012) 151.

[6] LHCв COLLABORATION collaboration, First observation and amplitude analysis of the $B^{-} \rightarrow D^{+} K^{-} \pi^{-}$decay, Phys. Rev. D 91 (2015) 092002.

[7] LHCв Collaboration collaboration, Amplitude analysis of $B^{0} \rightarrow \bar{D}^{0} K^{+} \pi^{-}$decays, Phys. Rev. D 92 (2015) 012012.

[8] LHCв Collaboration collaboration, Dalitz plot analysis of $B^{0} \rightarrow \bar{D}^{0} \pi^{+} \pi^{-}$decays, Phys. Rev. D 92 (2015) 032002.

[9] LHCв Collaboration collaboration, Amplitude analysis of $B^{-} \rightarrow D^{+} \pi^{-} \pi^{-}$decays, Phys. Rev. D 94 (2016) 072001.

[10] Particle Data Group collaboration, Review of particle physics, Phys. Rev. D 98 (2018) 030001.

[11] LHCв collaboration, Determination of quantum numbers for several excited charmed mesons observed in $B^{-} \rightarrow D^{*+} \pi^{-} \pi^{-}$decays, Phys. Rev. D101 (2020) 032005 [1911.05957].

[12] Fermilab E791 Collaboration collaboration, Model-independent measurement of S-wave $K^{-} \pi^{+}$systems using $D^{+} \rightarrow K \pi \pi$ decays from Fermilab E791, Phys. Rev. D 73 (2006) 032004. 
[13] The BaBar Collaboration collaboration, Measurement of the $I=1 / 2 K \pi \mathcal{S}$-wave amplitude from Dalitz plot analyses of $\eta_{c} \rightarrow K \bar{K} \pi$ in two-photon interactions, Phys. Rev. $D$ 93 (2016) 012005.

[14] Belle Collaboration collaboration, Study of $B^{-} \rightarrow D^{* * 0} \pi^{-}\left(D^{* * 0} \rightarrow D^{(*)+} \pi^{-}\right)$decays, Phys. Rev. D 69 (2004) 112002.

[15] LHCв Collaboration collaboration, Observation of Overlapping Spin-1 and Spin-3 $\bar{D}^{0} K^{-}$ Resonances at Mass $2.86 \mathrm{GeV} / c^{2}$, Phys. Rev. Lett. 113 (2014) 162001.

[16] LHCв Collaboration collaboration, Dalitz plot analysis of $B_{s}^{0} \rightarrow \bar{D}^{0} K^{-} \pi^{+}$decays, Phys. Rev. D 90 (2014) 072003. 